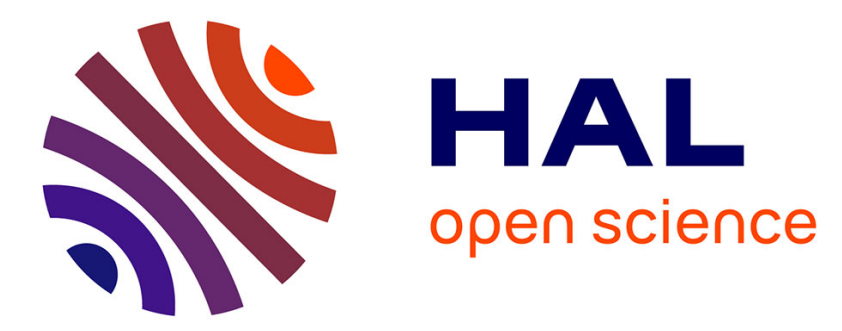

\title{
Investigating the phonological status of the Initial Accent in French: an Event-Related Potentials study
}

Noémie Ag Te Rietmolen, Radouane El Yagoubi, Robert Espesser, Cynthia Magnen, Corine Astésano

\section{- To cite this version:}

Noémie Ag Te Rietmolen, Radouane El Yagoubi, Robert Espesser, Cynthia Magnen, Corine Astésano. Investigating the phonological status of the Initial Accent in French: an Event-Related Potentials study. Speech Prosody 2016, May 2016, Boston, United States. 10.21437/SpeechProsody.2016-243 . hal-01577527

\section{HAL Id: hal-01577527 \\ https://hal.science/hal-01577527}

Submitted on 26 Aug 2017

HAL is a multi-disciplinary open access archive for the deposit and dissemination of scientific research documents, whether they are published or not. The documents may come from teaching and research institutions in France or abroad, or from public or private research centers.
L'archive ouverte pluridisciplinaire HAL, est destinée au dépôt et à la diffusion de documents scientifiques de niveau recherche, publiés ou non, émanant des établissements d'enseignement et de recherche français ou étrangers, des laboratoires publics ou privés. 


\section{Investigating the phonological status of the Initial Accent in French: an Event-Related Potentials study}

Conference Paper · June 2016

DOI: $10.21437 /$ SpeechProsody.2016-243

\section{CITATIONS}

2

5 authors, including:

\section{Radouane El Yagoubi}

University of Toulouse II - Le Mirail

20 PUBLICATIONS 244 CITATIONS

SEE PROFILE

\section{Corine Astésano}

University of Toulouse, France

53 PUBLICATIONS 450 CITATIONS

SEE PROFILE
READS

63

\section{Robert Espesser}

French National Centre for Scientific Research 105 PUBLICATIONS 1,019 CITATIONS

SEE PROFILE

Some of the authors of this publication are also working on these related projects:

Project

Project
COGNIPROS. Linguistic and cognitive evaluation of prosodic production and perception in deviant speech: stress phenomena. View project

PhonIACog - The role of the Initial Accent in prosodic structuring in French - From phonology to speech processing - View project 


\title{
Investigating the phonological status of the Initial Accent in French: an Event-Related Potentials study
}

\author{
Noémie te Rietmolen ${ }^{1}$, Radouane El Yagoubi ${ }^{2}$, Robert Espesser ${ }^{3}$, \\ Cynthia Magnen ${ }^{4}$, Corine Astésano ${ }^{1}$
}

\author{
${ }^{1}$ U.R.I Octogone-Lordat (E.A. 4156), Université de Toulouse, UTM, Toulouse, France \\ ${ }^{2}$ Laboratoire CLLE-LTC (UMR 5263), Université de Toulouse, UTM, Toulouse, France \\ ${ }^{3}$ Laboratoire Parole \& Langage (UMR 7309), Aix-Marseille Université, Aix-en-Provence, France \\ ${ }^{4}$ MSHS-T (USR 3414), Université de Toulouse, UTM, Toulouse, France \\ noemie.te-rietmolen@univ-tlse2.fr, yagoubi@univ-tlse2.fr, Robert.Espesser@lpl-aix.fr, \\ cynthia.magnen@univ-tlse2.fr, astesanoduniv-tlse2.fr
}

\begin{abstract}
This Event-Related Potentials (ERP) study investigates the use of prosodic information in the process of lexical access in French. In French, accentuation is said to be post-lexical, with a primary final accent (FA) and secondary initial accent (IA) marking the edges of the phrase. Results from previous studies, however, suggest IA may hold a demarcative function close to the level of the word. Still, the contribution of IA in word processing has not yet been empirically tested. In this study, participants listened to trisyllabic French nouns and pseudowords, with $(+\mathrm{IA})$ or without $(-\mathrm{IA})$ initial accent while completing a lexical decision task. We were mainly interested in modulations of the N325, a component assumed to reflect difficulties in the extraction of lexical stress patterns. ERP results show a larger N325 when stimuli were presented -IA, revealing both the automaticity of stress extraction and a preference for stress templates with initial accent.
\end{abstract}

Index Terms: stress, French, Initial Accent, lexical decision, Event-Related Potentials, N325

\section{Introduction}

The ability to understand spoken language is a fundamental and intriguing human skill. Considering speech is formed out of connected and co-articulated units with no spaces or other breaks, the manner in which we translate its signal into a sequence of words is far from obvious. One source that may help speech segmentation comes from the metrical structure of the signal. According to Metrical Segmentation Strategy (MSS), the segmentation of continuous speech is accomplished by relying on the dominant metrical pattern of the language [1]. In stress-based languages such as English and Dutch, where the vast majority of lexical words start with a strong syllable $[2,3]$, listeners are thought to exploit that high prosodic probability and initiate lexical access at each stressed syllable. But, while this may be a successful strategy in languages with lexical stress, segmenting on strong onsets is arguably much less efficient in languages in which the domain for metrical rules is not the lexical word.

French is often described as a syllable-based language with fairly homogeneous metrical weight on syllables. Consequently, it is held that the French metrical structure is defined by the syllable and that the syllable is used as the basic unit for segmenting speech $[4,5,6]$. This idea is further supported by the view that, in French, accentuation is post-lexical, demarcating boundaries not at the level of the word but at the level of groups of words. That is, the primary French accent, known as the final accent (FA), is fixed on the last syllable of the phrase, marking its right edge. When necessary (e.g. in case of long stretches of unaccented syllables), FA can be accompanied with a secondary initial accent (IA) that marks the left edge of the phrase [7]. So, French is considered a language without lexical stress, making accents unlikely candidates to cue lexical access.

In contrast with this view, Di Cristo's metrical model considers both FA and IA to be phonologically represented at the level of the prosodic word (i.e. close to the lexical word [8]) despite accentuation not being lexically distinctive in French [9]. According to this model, French accentuation thus provides not one, but two entries; at the left boundary and at the right boundary of the word. A number of studies investigating the use of French prosodic cues in word processing report results in line with Di Cristo's conjecture of (latent) stress templates underlying the representation of the prosodic word. Both the primary FA and the secondary IA have been found to guide French listeners in the segmentation of speech (for use of FA see $[10,11,12]$; for use of IA see $[13,14,15])$. These studies challenge the idea that French listeners adopt a syllable-based segmentation strategy (as proposed by [16]). They instead favor a strategy in which listeners rely on metrical stress patterns during speech comprehension. They, however, do not challenge the view that IA and FA demarcate phrase boundaries, and still consider accentuation to apply to the level of the Accentual Phrase (AP; [17]) and not to the level of the prosodic word. Assuming Di Cristo's view gives new perspectives on the speech segmentation strategy in French. Indeed, if French accentuation is actually a stress template encoded at the level close to the lexical word, IA and FA could readily notify listeners on when to initiate lexical access.

Here, we further investigate the representation of French accentuation and its contribution to word processing. More specifically, the status of the initial accent and its role in lexical access is examined. Because this accent is traditionally regarded as a secondary and optional accent, only complementary to the final accent, up until recently IA has received relatively little scientific attention. However, it been shown that, similar to FA, IA not only directs listeners in the segmentation of speech $[13,14,15]$, but also that IA is a more reliable cue in the marking of lexical structure than FA [18]. In the study, IA 
(a) -IA

(b) +IA

was shown to mark lower levels of structure, close to the lexical word. A later study on the perception of prominences indicated that IA is perceived as stronger than FA, in a manner independent from the depth of prosodic structure. This points towards an association between IA and word demarcation [19].

Following up on these results, a recent event-related potentials (ERP) study was carried out that lends further support to the notion of a phonological representation of IA [20]. In the study, Aguilera et al. investigated the phonological status of IA, using an oddball paradigm in which the presence of IA was manipulated on trisyllabic words. When presenting the oddball without IA, a clear MisMatch Negativity component (MMN) emerged. But, when the oddball was presented with IA, the resultant MMN was significantly smaller. The authors took this to indicate that IA is represented at the phonological longterm representation of the word and part of the French preferred stress template.

In the present ERP study, we sought to build on the MMN study and manipulated the presence of IA in a lexical decision task. We were particularly interested in modulations of the $\mathrm{N} 325$, a component assumed to reflect difficulties in the extraction of lexical stress templates [21]. The component was first encountered in a study in which the authors presented Dutch participants with a stress discrimination task. In the task, sequences of four bisyllabic words were presented with stress on the first syllable (the dominant metrical pattern in Dutch) or on the second syllable. Results showed that the less frequent stress template elicited a larger frontal negativity (the N325) than did the dominant stress template. This led the authors to conclude that the N325 may reflect the extraction of metrical stress during lexical access. If IA is linked to the phonological representation of prosodic words and is, along with FA, the expected stress template in French, presenting words without IA should elicit a larger N325 than presenting words with IA. To further attest for the pre-lexicality of stress encoding in French and question whether IA is part of the preferred metrical template, we test this metrical pattern on words and pseudowords.

\section{Methods}

\subsection{Speech stimuli}

The stimuli consisted of 120 trisyllabic French nouns (e.g. chocolat) and 120 trisyllabic pseudowords (e.g. chibute). The stimuli were extracted from sentences spoken by a naïve native speaker of French. In the sentences, the target words (lexical word or pseudoword) were placed at the beginning of a major phrase to increase the probability of clear IA and FA marking [18]. Stimuli with the most natural IA (+IA) were selected by a panel of three experts and re-synthesized without IA (-IA) using a customized quadratic algorithm in PRAAT [22].

Using the same algorithm as [20], the $f 0$ value of the first vowel (i.e. IA) was lowered near the $f 0$ value of the preceding (unaccented) determinant, to deaccentuate the first syllable (i.e. remove IA; see Figure 1). The algorithm progressively modified the $f 0$ values to reach the $f 0$ value at the beginning of the last (accented) vowel. This quadratic transformation allowed for micro-prosodic variations to be maintained, thus keeping the natural sound of the stimuli. The +IA stimuli were forward and back transformed to equalize the speech quality between +IA and -IA stimuli. The duration of the target words was held constant in both stress conditions (+IA; -IA), since only the $f 0$ parameter was manipulated (lexical words $m=813, s d=81$; pseudowords $m=844, s d=83$ ).

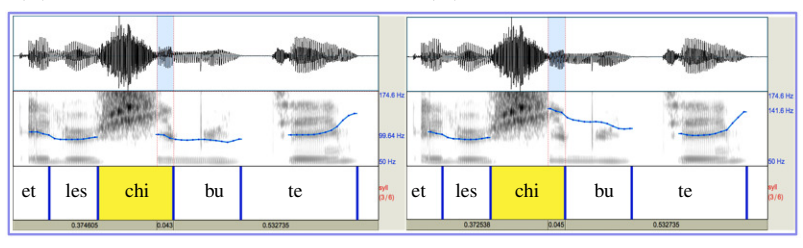

Figure 1: Example of $f 0$ resynthesis $(a)-I A$ and $(b)+I A$ on '[et les] chibute', with quadratic interpolation from the f0 value of the preceding determinant to the $f 0$ value at the beginning of the last stressed syllable for -IA targets.

\subsection{Participants}

26 French native speakers, aged 19 - 31 (mean age 25.4; 20 females), took part in the study. All subjects were right-handed, with normal hearing abilities and no reported history of neurological or language-related problems. Due to excessive artifacts in the EEG signal, 3 participants were excluded from analyses.

\subsection{Procedure}

Each participant was comfortably seated in an electrically shielded and sound attenuated room. Stimuli were presented through headphones and participants were allowed to adjust the volume to their individual preferences.

Participants were instructed to judge as quickly and accurately as possible whether a word was a real word or a pseudoword by pressing the left or right button on a button-box (button assignment was counter-balanced across participants). To ensure participants understood the task requirements, the experiment began with a short practice phase. This phase consisted of 12 trials that were very similar to the experimental trials, but were not included in the analyses.

Each participant listened to all 240 stimuli. Using Latin square designs, the four conditions (word +IA, word -IA, pseudoword +IA, pseudoword -IA) were evenly distributed over blocks, and block order was balanced between participants. In order to better control for eye-related EEG activity, each trial started with a $400 \mathrm{~ms}$ presentation of a white fixation cross at the center of a computer screen. The stimulus was presented immediately after the offset of the fixation cross. Participants were given a maximum of $2000 \mathrm{~ms}$ to give their answer. The intertrial interval (ITI) followed the participants' response and lasted until $2500 \mathrm{~ms}$ post stimulus onset. As a result, the duration of ITI varied, while trial duration was fixed at $2900 \mathrm{~ms}$. Total duration of the experiment, including the set-up of the EEG electrodes, was approximately $2 \mathrm{~h}$.

\subsection{EEG recording and preprocessing}

The EEG data were recorded with $32 \mathrm{Ag} / \mathrm{AgCl}$-sintered electrodes mounted on an elastic cap and located at standard left and right hemisphere positions over frontal, central, parietal, occipital and temporal areas (International 10/20 System; Jasper, 1958) at $\mathrm{Fz}, \mathrm{Cz}, \mathrm{Pz}, \mathrm{Oz}, \mathrm{Fp} 1, \mathrm{Fp} 2, \mathrm{AF} 3, \mathrm{~F} 3, \mathrm{AF} 4, \mathrm{~F} 4, \mathrm{C} 3, \mathrm{C} 4$, P3, P4, PO3, PO4, P5, P6, O1, O2, F7, F8, T3, T4, T5, T6, FC5, FC6, CP1, CP2, CP5 and CP6. To detect blinks and eyemovements, 4 additional electrodes were placed around the eyes (HEOG: bipolar channel placed lateral to the outer corner of both eyes; VEOG: bipolar channel placed above and below the left eye). The EEG and EOG signals were amplified by BioSemi amplifiers (ActiveTwo System) and digitized at $512 \mathrm{~Hz}$.

The data were preprocessed using the EEGLAB package [23] in Matlab [24]. Each electrode was re-referenced offline to 


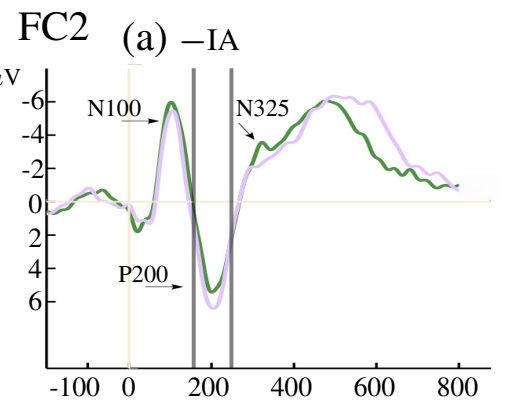

(b) + IA

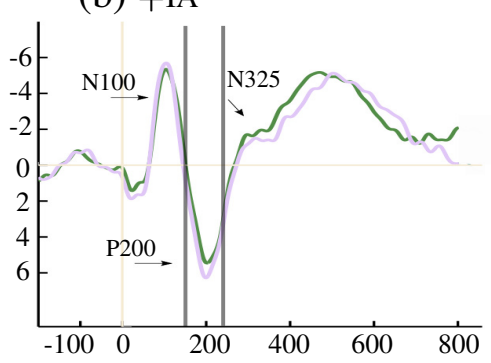

(c) main effect

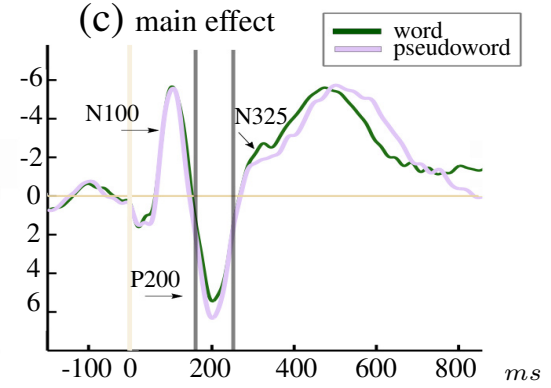

Figure 2: Grand average P200 in the lexical condition (word, pseudoword), recorded at the FC2 (frontocentral) electrode for: (a) -IA, (b) $+I A$, (c) main effect. The vertical gray bars indicate the selected P200 time window $(151-251 \mathrm{~ms})$. For ease of presentation, ERP waveforms are cut off at $800 \mathrm{~ms}$. Negativity is plotted as an upward deflection.

the algebraic average of the left and right mastoids. The data were band-pass filtered between $0.01-30 \mathrm{~Hz}$ and epoched from -0.2 to 2 seconds surrounding the onset of the speech signal. Following a visual inspection, epochs containing EMG or other artifacts not related to eye-movements or blinks were manually removed. Independent Components Analysis (ICA) was performed on the remaining epochs in order to identify and subtract components containing oculomotor artifacts from the data. Finally, data were averaged within and across participants to obtain the grand-averages for each of the 4 conditions.

\subsection{EEG analysis}

With its high temporal resolution, EEG provides a rich database to determine the exact latency of an effect. However, testing at all data points independently quickly leads to a multiple comparison problem where the risk of making Type I errors increases considerably. As EEG measures are not independent, but in fact temporally and spatially correlated, we used a nonparametric $t_{\max }$ permutation test to analyze the data [25, 26].

In $t_{\max }$ permutation testing, the null distribution is estimated by repeatedly resampling the obtained data and calculating $t$ scores for each sample. The most extreme $t$-scores $\left(t_{\max }\right)$ are selected for the null distribution. Finally, the $t$-scores of the observed data are computed and compared to the simulated $t_{\max }$ distribution, just as in parametric hypothesis testing.

As with each permutation the chance of obtaining a large $t_{\max }$ increases, the test automatically becomes more conservative when making more comparisons. Also, since the actual, obtained data is used to estimate the null distribution, the test does not assume test independence, allowing for stringent control of Type I error without considerable decrease in sensitivity. To further maximize power and reduce the number of comparisons, the data were down-sampled to $125 \mathrm{~Hz}$ and time-windows were estimated following the method used in [21]. We were mainly interested in modulations of the P200 (151 - $251 \mathrm{~ms})$ and the N325 (201- $431 \mathrm{~ms})$, as these two components reflect auditory processes in the pre-lexical stage of word processing (acoustical processing and stress extraction, respectively [27] [21]).

Each comparison of interest was analyzed with a separate repeated measures, two-tailed $t$-tests, using the original data and 2500 random permutations to approximate the null distribution for the customary family-wise alpha $(\alpha)$ level of $0.05^{1}$.

\footnotetext{
${ }^{1}$ In fact we used more than twice the number of permutations Manly suggested for an alpha at $5 \%$.[28]
}

\section{Results}

\subsection{Behavioral results}

Behavioral data (error rates and reaction times) were analyzed with paired two-tailed $t$-tests in $\mathrm{R}$ [29]. Overall, performance on the lexical decision task revealed high accuracy $(<5 \%$ errors) with no differences between conditions. Reaction times showed a main effect of lexicality $(t=-16.85, p<0.001)$; words were responded to faster than pseudowords. Presence of IA had no effect on response latencies $(p=0.7, n s)$.

\subsection{ERP results}

In the P200 time-window (Figure 2), there was a main effect of lexicality (critical $t$-score: $\pm 3.5589, p<0.05$ ). Pseudowords elicited a larger P200 than words in the frontocentral region (FC2) peaking $182 \mathrm{~ms}$ after stimulus presentation. The difference between words and pseudowords was also significant within the condition without IA (critical $t$-score: \pm 3.575 , $p<0.05)$. Within the condition with IA this effect was not significant $(p=0.4, n s)$.

In the N325 time-window (Figure 3), there was a main effect of presence of IA (critical $t$-score: $\pm 3.6887, p<0.05$ ). Compared to stimuli + IA, stimuli - IA elicited a larger negativity in the frontocentral region $(\mathrm{FC} 2$ and $\mathrm{Cz}$ ) from $318-358$ ms after stimulus presentation. The difference in ERP amplitude is small, but robust and comparable to the amplitude difference reported in Böcker et al $(1-2.5 \mu \mathrm{V})$. The effect was also significant within the lexical words condition (critical $t$-score: $\pm 3.8546, p<0.05$ ); words -IA resulted in a larger negativity than words + IA. There was a similar trend in the pseudowords condition, although it did not reach significance $(p=0.09, n s)$. A visual inspection of the ERPs, however, suggests similar processes between words and pseudowords.

\section{Discussion}

In the present study, we examined the interplay between accentuation and lexical access in French. We were particularly interested in the status and possible roles of the initial accent. Our results show that IA is represented in the French preferred and expected stress template. As pre-lexical language-specific stress templates are suggested to serve as gateways to the mental lexicon, IA could thus play an important role in the process of speech segmentation in French.

We used a lexical decision task in which we manipulated the presence of IA. The manipulation modulated the resultant frontocentral N325; a larger N325 emerged when IA had been 

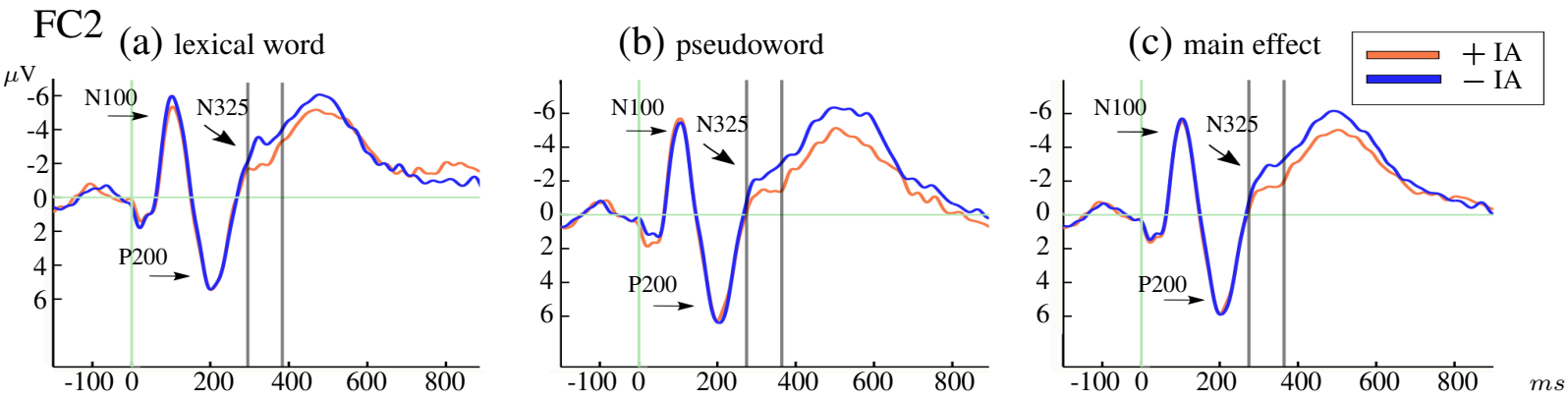

Figure 3: Grand average N325 in the $\pm I A$ condition, recorded at the FC2 (frontocentral) electrode for: (a) lexical words, (b) pseudowords, (c) main effect.The vertical gray bars indicate the selected time window (201 - $431 \mathrm{~ms})$. For ease of presentation, ERP waveforms are cut off at $800 \mathrm{~ms}$. Negativity is plotted as an upward deflection.

omitted. As the N325 is assumed to reflect difficulties in the extraction of lexical stress, this result indicates a stress processing cost when stimuli are presented without IA. Recall that Böcker et al. report similar findings after manipulating stress in Dutch, a language with lexical stress [21]. In their study, listeners were asked to discriminate between the Dutch dominant stress template and a less frequent stress template. Words presented without the dominant stress pattern elicited a more ample N325. In our study, words presented without IA resulted in the larger N325 suggesting that, even though stress is not lexically distinctive in French, IA is part of the French expected stress pattern ( $c f[20])$. In addition, while in the study of Böcker et al. participants were asked to explicitly attend the metrical structure of the stimuli, in the present study, attention was diverted from the stress manipulation using a lexical decision task. Still finding a robust modulation of the N325, further demonstrates that word processing naturally engaged the pre-lexical extraction of IA. That is, we show that lexical access is facilitated when words are presented with the French preferred stress template, i.e. with the initial accent.

The amplitude modulation of the N325 was small (between $1-2.5 \mu \mathrm{V}$ ), but robust as revealed by our conservative nonparametric statistics (see Methods section). In fact, finding a relatively small difference in amplitude was expected and comparable to the amplitude difference in Böcker et al. [21]. Similar to Böcker et al., we did not manipulate the legality, but rather the probability of the presented stress templates. That is, while in French there is a preference for words marked with IA, words without IA are not illegal. Indeed, in continuous speech IA is not always realized and may be suppressed to serve for instance a more rhythmically balancing function. So, while French listeners may expect and prefer words to be marked with IA, words without IA do not exceedingly hamper word processing.

Our manipulation of IA did not modulate the P200, a component thought to reflect the bottom-up extraction of purely physical/acoustical parameters [27]. This indicates that our results reveal a more controlled process in which stress is extracted in a top-down fashion. We did find lexicality to affect the P200 when stimuli were presented without IA; pseudowords elicited a more ample P200 than did words when presented without IA. This effect is surprising since the latency range of the P200 precedes lexical processing [30]. Considering the location of the P200 (frontocentral; similar to the location of the N325) the effect appears to be the product of a temporal overlap between the P200 and the N325. The N325 was more negative for -IA stimuli than +IA stimuli and this difference was larger in the words condition than in the pseudowords condition. This means that in the -IA condition the overlap between the P200 and N325 will be more evident for words than for pseudowords, while in the +IA condition the overlap will be smaller (as +IA stimuli elicited a smaller N325). In fact, Böcker et al. report a similar overlap between the N325 and the P200 at the frontocentral electrodes. Finding an overlap between the N325 and the P200 implies that the process of stress extraction starts before our predefined N325 time-window $(201-431 \mathrm{~ms})$ and during the P200 time-window $(151-251 \mathrm{~ms})$. Such an early latency confirms that lexical access crucially involved an automatic, pre-lexical extraction of the French initial accent.

A visual inspection of the ERP components suggests \pm IA also affected the later integration stages of word processing, as there seems to be an amplitude difference in the latency range typically associated with the N400 [31]. It is however unlikely that these late amplitude modulations really reflect difficulties in the post-lexical process of semantic integration, as word were presented in isolation. Moreover, the N400 is typically maximal over centroparietal sites [31,32], while the reported ERPs in the current study have a frontocentral distribution. A more probable explanation is suggested by Böcker et al., who encountered a similar late frontocentral amplitude difference and interpret it to reflect N325 residue. A study to determine if \pm IA also affects the later stages of speech processing, is currently in progress. In the study, \pm IA words are embedded within a congruent or incongruent semantic context, and as such, the study is better adapted to give insight into whether IA also affects the later stages of speech processing.

\section{Conclusions}

In this study, we investigated the status of the French initial accent. Our ERP results demonstrate that IA is linked to the phonological representation of words. Words presented without initial accent elicited a more ample N325, a component that indexes difficulties in pre-lexical stress extraction. Moreover, as we diverted attention away from our stress manipulation with a lexical decision task, the extraction of IA seems to be an automatic step during the early stages of word processing. This indicates that the initial accent is part of the French preferred stress template and as such, contrary to popular belief, plays a valuable role in French speech comprehension.

\section{Acknowledgments}

This study is supported by the Agence Nationale de la Recherche grant ANR-12-BSH2-0001 (PI: Corine Astésano) 


\section{References}

[1] A. Cutler and D. Norris, "The role of strong syllables in segmentation for lexical access.” J. Exp. Psychol. Hum. Percept. Perform., vol. 14, no. 1, pp. 113-121, 1988.

[2] A. Cutler and D. M. Carter, "The predominance of strong initial syllables in the English vocabulary," Comput. Speech Lang., vol. 2, no. 3-4, pp. 133-142, 1987.

[3] J. Vroomen and B. De Gelder, "Metrical segmentation and lexical inhibition in spoken word recognition." Journal of Experimental Psychology: Human perception and performance, vol. 21, no. 1, p. $98,1995$.

[4] J. Mehler and R. Hayes, "The role of syllables in speech processing: Infant and adult data [and discussion]," Philosophical Transactions of the Royal Society B: Biological Sciences, vol. 295, no. 1077, pp. 333-352, 1981

[5] J. Mehler, J. Y. Dommergues, U. Frauenfelder, and J. Segui, “The syllable's role in speech segmentation," Journal of verbal learning and verbal behavior, vol. 20, no. 3, pp. 298-305, 1981.

[6] A. Cutler, J. Mehler, D. Norris, and J. Segui, “The syllable's differing role in the segmentation of french and english," Journal of memory and language, vol. 25, no. 4, pp. 385-400, 1986.

[7] M. Rossi, L'intonation: le système du français: description et modélisation. Editions Ophrys, 1999.

[8] E. Selkirk, "The prosodic structure of function words," Signal to syntax: Bootstrapping from speech to grammar in early acquisition, vol. 187, p. 214, 1996

[9] A. Di Cristo, "Vers une modélisation de l'accentuation du français (seconde partie)," Journal of French Language Studies, vol. 10, no. 01, pp. 27-44, 2000 .

[10] M.-H. Banel and N. Bacri, "On metrical patterns and lexical parsing in french," Speech Communication, vol. 15, no. 1, pp. 115126, 1994.

[11] O. Bagou, C. Fougeron, and U. H. Frauenfelder, "Contribution of prosody to the segmentation and storage of" words" in the acquisition of a new mini-language," in Speech Prosody 2002, International Conference, 2002.

[12] A. Christophe, S. Peperkamp, C. Pallier, E. Block, and J. Mehler, "Phonological phrase boundaries constrain lexical access i. adult data," Journal of Memory and Language, vol. 51, no. 4, pp. 523 547, 2004.

[13] P. Welby, "The role of early fundamental frequency rises and elbows in french word segmentation," Speech Communication, vol. 49, no. 1, pp. 28-48, 2007.

[14] E. Spinelli, P. Welby, and A.-L. Schaegis, "Fine-grained access to targets and competitors in phonemically identical spoken sequences: the case of french elision," Language and cognitive processes, vol. 22, no. 6, pp. 828-859, 2007.

[15] E. Spinelli, N. Grimault, F. Meunier, and P. Welby, "An intonational cue to word segmentation in phonemically identical sequences," Attention, Perception, \& Psychophysics, vol. 72, no. 3, pp. 775-787, 2010

[16] A. Content, R. K. Kearns, and U. H. Frauenfelder, "Boundaries versus onsets in syllabic segmentation," Journal of Memory and Language, vol. 45, no. 2, pp. 177-199, 2001.

[17] S.-A. Jun and C. Fougeron, "A phonological model of french intonation," in Intonation. Springer, 2000, pp. 209-242.

[18] C. Astésano, E. G. Bard, and A. Turk, "Structural influences on initial accent placement in French." Lang. Speech, vol. 50, no. Pt 3, pp. 423-446, 2007.

[19] C. Astésano, R. Bertrand, R. Espesser, and N. Nguyen, "Perception des frontières et des proéminences en français," JEP-TALNRECITAL, 2012

[20] M. Aguilera, R. El Yagoubi, R. Espesser, and C. Astésano, "Event-Related Potential of Initial Accent Processing in French," Speech Prosody 2014, no. Umr 5263, pp. 3-7, 2014.
[21] K. B. Böcker, M. Bastiaansen, J. Vroomen, C. H. Brunia, and B. Gelder, "An erp correlate of metrical stress in spoken word recognition," Psychophysiology, vol. 36, no. 6, pp. 706-720, 1999.

[22] P. Boersma and D. Weenink, " $\{\mathrm{P}\}$ raat: doing phonetics by computer," 2010.

[23] A. Delorme and S. Makeig, "Eeglab: an open source toolbox for analysis of single-trial eeg dynamics including independent component analysis," Journal of neuroscience methods, vol. 134 no. 1, pp. 9-21, 2004.

[24] T. MathWorks, "Image processing toolboxtm user's guide, version r2014a," The MathWorks, 2014.

[25] D. M. Groppe, T. P. Urbach, and M. Kutas, "Mass univariate analysis of event-related brain potentials/fields i: A critical tutorial review," Psychophysiology, vol. 48, no. 12, pp. 1711-1725, 2011.

[26] S. J. Luck, An introduction to the event-related potential technique. MIT press, 2014.

[27] S. A. Hillyard and T. W. Picton, "Electrophysiology of cognition," Comprehensive Physiology, 1987.

[28] B. Manly, "Randomization, bootstrap, and monte carlo methods in biologychapman and hall," New York, 1997.

[29] R. C. Team, "The r project for statistical computing," $R$ Foundation for Statistical Computing web-site. www. R-project. org. Accessed June, vol. 9, 2014

[30] F. Grosjean, "Spoken word recognition processes and the gating paradigm," Perception \& Psychophysics, vol. 28, no. 4, pp. 267$283,1980$.

[31] C. Brown and P. Hagoort, "The processing nature of the n400: Evidence from masked priming," Journal of Cognitive Neuroscience, vol. 5, no. 1, pp. 34-44, 1993.

[32] M. Kutas and K. D. Federmeier, "Electrophysiology reveals semantic memory use in language comprehension," Trends in cognitive sciences, vol. 4, no. 12, pp. 463-470, 2000. 